\title{
Oscillation of Second Order Nonlinear Neutral Differential Equations with Mixed Neutral Term
}

\section{Ramalingam Arul*, Venkatachalam Subramaniyam Shobha}

Department of Mathematics, Kandaswami Kandar's College, Velur, India Email: ${ }^{*}$ rarulkkc@gmail.com

Received 5 June 2015; accepted 4 September 2015; published 7 September 2015

Copyright @ 2015 by authors and Scientific Research Publishing Inc.

This work is licensed under the Creative Commons Attribution International License (CC BY). http://creativecommons.org/licenses/by/4.0/

(c) (i) Open Access

\section{Abstract}

In this paper, we obtained some sufficient conditions for the oscillation of all solutions of the second order neutral differential equation of the form $\left(r(t) z^{\prime}(t)\right)^{\prime}+q(t) f(x(\sigma(t)))=0, t \geq t_{0} \geq 0$ where $z(t)=x(t)+a(t) x(t-\tau)+b(t) x(t+\delta)$, and $\int_{t_{0}}^{\infty} \frac{1}{r(t)} d t<\infty$. Examples are provided to illustrate the main results.

Keywords

Second Order, Nonlinear Differential Equation, Mixed Neutral Term, Oscillation

\section{Introduction}

In this paper, we are concerned with the oscillatory behavior of solutions of the second order nonlinear neutral differential equation of the form

$$
\left(r(t) z^{\prime}(t)\right)^{\prime}+q(t) f(x(\sigma(t)))=0, t \geq t_{0} \geq 0
$$

where $z(t)=x(t)+a(t) x(t-\tau)+b(t) x(t+\delta)$, subject to the following conditions:

$\left(C_{1}\right) a, b, q \in C\left(\left[t_{0}, \infty\right), \mathbb{R}\right), 0 \leq a(t) \leq a<\infty, 0 \leq b(t) \leq b<\infty$, and $q(t)>0$ for all $t \geq t_{0}$;

\footnotetext{
${ }^{*}$ Corresponding author.
}

How to cite this paper: Arul, R. and Shobha, V.S. (2015) Oscillation of Second Order Nonlinear Neutral Differential Equations with Mixed Neutral Term. Journal of Applied Mathematics and Physics, 3, 1080-1089. 
$\left(C_{2}\right) \quad r \in C\left(\left[t_{0}, \infty\right), \mathbb{R}\right), r(t)>0$, and $\int_{t_{0}}^{\infty} \frac{1}{r(t)} \mathrm{d} t<\infty$;

$\left(C_{3}\right) \tau, \delta$ are nonnegative constants, $\sigma \in C\left(\left[t_{0}, \infty\right), \mathbb{R}\right), \sigma^{\prime}(t)>0, \sigma(t) \leq t, \lim _{t \rightarrow \infty} \sigma(t)=\infty$, and $\sigma(t \pm \alpha)=\sigma(t) \pm \alpha$ for any $\alpha>0$;

$\left(C_{4}\right) \quad f \in C(\mathbb{R}, \mathbb{R}), \frac{f(u)}{u} \geq k>0$ for $u \neq, k$ is a constant.

By a solution of Equation (1), we mean a continuous function $x$ defined on an interval $\left[t_{x}, \infty\right)$ such that $r(t) z^{\prime}(t)$ is continuously differentiable and $x$ satisfies Equation (1) for all $t \in\left[t_{x}, \infty\right)$. We consider only solutions satisfying condition $\sup \left\{|x(t)|: t \geq T \geq t_{x}\right\}>0$, and tacitly assume that Equation (1) possess such solutions. As usual, a solution of Equation (1) is called oscillatory if it is neither eventually positive nor eventually negative; otherwise we call it nonosicllatory.

From the literature, it is known that second order neutral functional differential equations have applications in problems dealing with vibrating masses attached to an elastic bar and in some variational problems. For further applications and questions regarding existence and uniqueness of solutions of neutral functional differential equations, see [1]-[3].

In recent years, there has been an increasing interest in establishing conditions for the oscillation or nonoscillation of solution of neutral functional differential equations, see [4]-[20] for example, and the references cited therein.

In [21], Xu and Meng obtained some sufficient conditions which guarantees that every solution $x$ of equation (1) when $b(t) \equiv 0$, oscillates or $\lim _{t \rightarrow \infty} x(t)=0$.

Ye and $\mathrm{Xu}$ [22] studied equation when $b(t) \equiv 0$, and established some new oscillation criteria for Equation (1).

In [23], Han et al. considered Equation (1) with $b(t) \equiv 0$ and $0 \leq a(t) \leq 1$, and obtained some sufficient conditions which ensure that every solution of Equation (1) is oscillatory.

In [24], the present authors established some sufficient conditions for the oscillation of all solutions of

Equation (1) when $\int_{t_{0}}^{\infty} \frac{1}{r(t)} \mathrm{d} t=\infty$. Therefore in this paper we try to obtain some new oscillation criteria for

Equation (1). In Section 2, we use Riccati transformation technique to obtain some sufficient conditions for the oscillation of all solutions of Equation (1). Examples are provided in Section 3 to illustrate the main results.

\section{Oscillation Results}

In this section, we obtain some new oscillation criteria for the Equation (1). We begin with the following theorem.

Theorem 2.1 If

$$
\int_{t_{0}}^{\infty} Q(t) \mathrm{d} t=\infty
$$

and

$$
\limsup _{t \rightarrow \infty} \int_{t_{0}}^{t}\left[k Q(s) \delta^{n}(s)-\frac{n^{2}(1+a+b)}{4 r(s) \delta^{2-n}(s)}\right] \mathrm{d} s=\infty
$$

where $n \geq 1, Q(t)=\min \{q(t), q(t-\tau), q(t+\delta)\}$, and $\delta(t)=\int_{t}^{\infty} \frac{1}{r(s)} \mathrm{d}$ then every solution of Equation (1) is oscillatory.

Proof. Suppose that $x(t)$ is a nonsocillatory solution of Equation (1). Without loss of generality, we may assume that there exists $t_{1} \geq t_{0}$ such that. $x(t)>0, x(t-\tau)>0, x(t+\delta)>0$ and $x(\sigma(t))>0$ for all $t \geq t_{1}$. From the definition of $z(t)$, we have $z(t)>0$, and from Equation (1), $r(t) z^{\prime}(t)$ is nonincreasing eventually. Hence, it is easy to conclude that there exist two possible cases of the sign of $z^{\prime}(t)$, that is, $z^{\prime}(t)>0$ or $z^{\prime}(t)<0$ for all $t \geq t_{2} \geq t_{1}$. 
First assume that $z^{\prime}(t)>0$ for all $t \geq t_{2}$. From the Equation (1), we have

$$
\begin{aligned}
& \left(r(t) z^{\prime}(t)\right)^{\prime}+k q(t) x(\sigma(t))+a\left(r(t-\tau) z^{\prime}(t-\tau)\right)^{\prime}+a k q(t-\tau) x(\sigma(t-\tau)) \\
& +b\left(r(t+\delta) z^{\prime}(t+\delta)\right)^{\prime}+b k q(t+\delta) x(\sigma(t+\delta)) \leq 0
\end{aligned}
$$

or

$$
\left(r(t) z^{\prime}(t)\right)^{\prime}+a\left(r(t-\tau) z^{\prime}(t-\tau)\right)^{\prime}+b\left(r(t+\delta) z^{\prime}(t+\delta)\right)^{\prime}+k Q(t) z(\sigma(t)) \leq 0, t \geq t_{2} .
$$

Integrating (4) from $t_{2}$ to $t$ and using the fact $z(t) \geq c>0$ for $t \geq t_{2}$, we obtain

$$
\int_{t_{2}}^{t} Q(s) \mathrm{d} s<\infty
$$

a contradiction to (2.1).

If $z^{\prime}(t)<0$, then we define the function $w$ by

$$
w(t)=\frac{r(t) z^{\prime}(t)}{z(t)}, t \geq t_{2} .
$$

Clearly $w(t)<0$. Nothing that $r(t) z^{\prime}(t)$ is nonincreasing, we obtain

$$
r(s) z^{\prime}(s) \leq r^{\prime}(t) z^{\prime}(t), s \geq t \geq t_{2} .
$$

Dividing the last inequality by $r(s)$ and integrating it from $t$ to $\ell$, we obtain

$$
z(\ell) \leq z(t)+r(t) z^{\prime}(t) \int_{t}^{\ell} \frac{\mathrm{d} s}{r(s)}, \ell \geq t \geq t_{2} .
$$

Letting $\ell \rightarrow \infty$ in the last inequality, we see that

$$
0 \leq z(t)+r(t) z^{\prime}(t) \delta(t), t \geq t_{2} .
$$

Therefore,

$$
\frac{r(t) z^{\prime}(t)}{z(t)} \delta(t) \geq-1, t \geq t_{2} .
$$

From (5), we have

$$
-1 \leq w(t) \delta(t) \leq 0, t \geq t_{2} .
$$

Next, we introduce another function $u$ by

$$
u(t)=\frac{r(t-\tau) z^{\prime}(t-\tau)}{z(t)}, t \geq t_{2} .
$$

Clearly $u(t)<0$. Noting that $r(t) z^{\prime}(t)$ is nonincreasing, we have $r(t-\tau) z^{\prime}(t-\tau) \geq r(t) z^{\prime}(t)$. Then, $u(t) \geq w(t)$. From (6), we obtain

$$
-1 \leq u(t) \delta(t) \leq 0, t \geq t_{2}
$$

Similarly, we introduce another function $v$ by

$$
v(t)=\frac{r(t+\delta) z^{\prime}(t+\delta)}{z(t)}, t \geq t_{2} .
$$

Clearly $v(t)<0$. Since $r(t) z^{\prime}(t)$ is nonincreasing, we have

$$
r(s) z^{\prime}(s) \leq r(t+\delta) z^{\prime}(t+\delta), s \geq t+\delta \geq t_{2} .
$$

Dividing the last inequality by $r(s)$ and integrating it from $t$ to $\ell$, we obtain 


$$
z(\ell) \leq z(t)+r(t+\delta) z^{\prime}(t+\delta) \int_{t}^{\ell} \frac{1}{r(s)} \mathrm{d} s, \ell \geq t+\delta \geq t_{2} .
$$

Letting $\ell \rightarrow \infty$, we see that

$$
-1 \leq v(t) \delta(t) \leq 0, t+\delta \geq t_{2} .
$$

Differentiating (5), we obtain

$$
w^{\prime}(t) \leq \frac{\left(r(t) z^{\prime}(t)\right)^{\prime}}{z(t)}-\frac{w^{2}(t)}{r(t)}, t \geq t_{3} \geq t_{2}+\delta .
$$

Differentiating (8), we have

$$
u^{\prime}(t) \leq \frac{\left(r(t-\tau) z^{\prime}(t-\tau)\right)^{\prime}}{z(t)}-\frac{u^{2}(t)}{r(t)}, t \geq t_{3}
$$

Differentiating (10), we have

$$
v^{\prime}(t) \leq \frac{\left(r(t+\delta) z^{\prime}(t+\delta)\right)^{\prime}}{z(t)}-\frac{v^{2}(t)}{r(t)}, t \geq t_{3} .
$$

Inview of (12), (13) and (14), we can obtain

$$
\begin{aligned}
w^{\prime}(t)+a u^{\prime}(t)+b v^{\prime}(t) \leq & \frac{\left(r(t) z^{\prime}(t)\right)^{\prime}}{z(t)}+a \frac{\left(r(t-\tau) z^{\prime}(t-\tau)\right)^{\prime}}{z(t)}+b \frac{\left(r(t+\delta) z^{\prime}(t+\delta)\right)^{\prime}}{z(t)} \\
& -\frac{w^{2}(t)}{r(t)}-a \frac{u^{2}(t)}{r(t)}-b \frac{v^{2}(t)}{r(t)}, t \geq t_{3} .
\end{aligned}
$$

From (4) and (15), we obtain

$$
w^{\prime}(t)+a u^{\prime}(t)+b v^{\prime}(t) \leq-k Q(t)-\frac{w^{2}(t)}{r(t)}-a \frac{u^{2}(t)}{r(t)}-b \frac{v^{2}(t)}{r(t)}, t \geq t_{3} .
$$

Multiplying (16) by $\delta^{n}(t)$ and integrating from $t_{3}$ to $t$, we have

$$
\begin{aligned}
& \delta^{n}(t) w(t)-\delta^{n}\left(t_{3}\right) w\left(t_{3}\right)+n \int_{t_{3}}^{t} \frac{w(s) \delta^{n-1}(s)}{r(s)} \mathrm{d} s+n \int_{t_{3}}^{t} \frac{w^{2}(s) \delta^{n}(s)}{r(s)} \mathrm{d} s+a \delta^{n}(t) u(t) \\
& -a \delta^{n}\left(t_{3}\right) u\left(t_{3}\right)+a n \int_{t_{3}}^{t} \frac{u(s) \delta^{n-1}(s)}{r(s)} \mathrm{d} s+a n \int_{t_{3}}^{t} \frac{u^{2}(s) \delta^{n}(s)}{r(s)} \mathrm{d} s+b \delta^{n}(t) v(t) \\
& -b \delta^{n}\left(t_{3}\right) v\left(t_{3}\right)+b n \int_{t_{3}}^{t} \frac{v(s) \delta^{n-1}(s)}{r(s)} \mathrm{d} s+b \int_{t_{3}}^{t} \frac{v^{2}(s) \delta^{n}(s)}{r(s)} \mathrm{d} s+k \int_{t_{3}}^{t} \delta^{n}(s) Q(s) \mathrm{d} s \leq 0 .
\end{aligned}
$$

From the above inequality, we obtain

$$
\begin{aligned}
& \delta^{n}(t) w(t)-\delta^{n}\left(t_{3}\right) w\left(t_{3}\right)+a \delta^{n}(t) u(t)-a \delta^{n}\left(t_{3}\right) u\left(t_{3}\right)+b \delta^{n}(t) v(t)-b \delta^{n}\left(t_{3}\right) v\left(t_{3}\right) \\
& +k \int_{t_{3}}^{t} \delta^{n}(s) Q(s) \mathrm{d} s-n^{2} \frac{(1+a+b)}{4} \int_{t_{3}}^{t} \frac{\mathrm{d} s}{r(s) \delta^{n-2}(s)} \leq 0 .
\end{aligned}
$$

Thus, it follows that

$$
\begin{aligned}
& \delta^{n}(t) w(t)+a \delta^{n}(t) u(t)+b \delta^{n}(t) v(t)+\int_{t_{3}}^{t}\left[k \delta^{n}(s) Q(s)-n^{2} \frac{1+a+b}{4 r(s) \delta^{2-n}(s)}\right] \mathrm{d} s \\
& \leq \delta^{n}\left(t_{3}\right) w\left(t_{3}\right)+a \delta^{n}\left(t_{3}\right) u\left(t_{3}\right)+b \delta^{n}\left(t_{3}\right) v\left(t_{3}\right) .
\end{aligned}
$$


By (7), (9) and (11), we obtain that

$$
\int_{t_{3}}^{t}\left[k \delta^{n}(s) Q(s)-n^{2} \frac{(1+a+b)}{4 r(s) \delta^{2-n}(s)}\right] \mathrm{d} s \leq \delta^{n-1}\left(t_{3}\right)(1+a+b)+\delta^{n}\left(t_{3}\right)\left(w\left(t_{3}\right)+a u\left(t_{3}\right)+b v\left(t_{3}\right)\right)
$$

which contradicts (3). The proof is now complete.

Corollary 2.1. Assume that $\sigma(t)=t-\tau$ with $\sigma \geq \tau$ for $t \geq t_{0}$. Further assume that (2.1) and (3) hold. Then every solution of Equation (1) is oscillatory.

Proof. The proof follows from Theorem 2.1.

Theorem 2.2. Assume that $\sigma(t) \leq t-\tau$ for $t \geq t_{0}$. If condition (2.1) holds and

$$
\limsup _{t \rightarrow \infty} \int_{t_{0}}^{t} \delta^{2}(s) Q(s) \mathrm{d} s=\infty
$$

then every solution of Equation (1) is oscillatory.

Proof. Let $x(t)$ be a nonsocillatory solution of Equation (1). Without loss of generality, we may assume that there exists $t_{1} \geq t_{0}$ such that $x(t)>0, x(t-\tau)>0, x(t+\delta)>0$ and $x(\sigma(t))>0$ for all $t \geq t_{1}$. By equation (1), $r(t) z^{\prime}(t)$ is nonincreasing eventually. Hence, it is easy to conclude that there exist two possible cases of the sign of $z^{\prime}(t)$, that is, $z^{\prime}(t)>0$ or $z^{\prime}(t)<0$ for all $t \geq t_{2} \geq t_{1}$. If $z^{\prime}(t)>0$, then we are back to the case of Theorem 2.1, and we can obtain a contradiction to (2.1). If $z^{\prime}(t)<0$, then we define $w, u$ and $v$ as in Theorem 2.1. Then proceed as in the proof of Theorem 2.1, we obtain (7), (9), (11) and (16) for $t \geq t_{3} \geq t_{2}$. Multiplying (16) by $\delta^{2}(t)$ and integrating from $t_{3}$ to $t$ yields

$$
\begin{aligned}
& \delta^{2}(t) w(t)-\delta^{2}\left(t_{3}\right) w\left(t_{3}\right)+2 \int_{t_{3}}^{t} \frac{w(s) \delta(s)}{r(s)} \mathrm{d} s+\int_{t_{3}}^{t} \frac{w^{2}(s) \delta^{2}(s)}{r(s)} d s+a \delta^{2}(t) u(t) \\
& -a \delta^{2}\left(t_{3}\right) u\left(t_{3}\right)+2 a \int_{t_{3}}^{t} \frac{u(s) \delta(s)}{r(s)} \mathrm{d} s+a \int_{t_{3}}^{t} \frac{u^{2}(s) \delta^{2}(s)}{r(s)} \mathrm{d} s+b \delta^{2}(t) v(t) \\
& -b \delta^{2}\left(t_{3}\right) v\left(t_{3}\right)+2 b \int_{t_{3}}^{t} \frac{v(s) \delta(s)}{r(s)} d s+b \int_{t_{3}}^{t} \frac{v^{2}(s) \delta^{2}(s)}{r(s)} d s+k \int_{t_{3}}^{t} \delta^{2}(s) Q(s) \mathrm{d} s \leq 0 .
\end{aligned}
$$

It follows from $\left(\mathrm{C}_{2}\right)$ and (7) that

$$
\begin{gathered}
\left|\int_{t_{3}}^{\infty} \frac{w(s) \delta(s)}{r(s)} d s\right| \leq \int_{t_{3}}^{\infty} \frac{|w(s) \delta(s)|}{r(s)} \mathrm{d} s \leq \int_{t_{3}}^{\infty} \frac{1}{r(s)} \mathrm{d} s<\infty, \\
\int_{t_{3}}^{t} \frac{w^{2}(s) \delta^{2}(s)}{r(s)} \mathrm{d} s \leq \int_{t_{3}}^{\infty} \frac{1}{r(s)} \mathrm{d} s<\infty .
\end{gathered}
$$

Inview of (9), we have

$$
\begin{gathered}
\left|\int_{t_{3}}^{\infty} \frac{u(s) \delta(s)}{r(s)} \mathrm{d} s\right| \leq \int_{t_{3}}^{\infty} \frac{|u(s) \delta(s)|}{r(s)} \mathrm{d} s \leq \int_{t_{3}}^{\infty} \frac{1}{r(s)} \mathrm{d} s<\infty, \\
\int_{t_{3}}^{t} \frac{u^{2}(s) \delta^{2}(s)}{r(s)} \mathrm{d} s \leq \int_{t_{3}}^{\infty} \frac{1}{r(s)} \mathrm{d} s<\infty .
\end{gathered}
$$

From (11), we obtain

$$
\begin{gathered}
\left|\int_{t_{3}}^{\infty} \frac{v(s) \delta(s)}{r(s)} \mathrm{d} s\right| \leq \int_{t_{3}}^{\infty} \frac{|v(s) \delta(s)|}{r(s)} \mathrm{d} s \leq \int_{t_{3}}^{\infty} \frac{1}{r(s)} \mathrm{d} s<\infty, \\
\int_{t_{3}}^{t} \frac{v^{2}(s) \delta^{2}(s)}{r(s)} \mathrm{d} s \leq \int_{t_{3}}^{\infty} \frac{1}{r(s)} \mathrm{d} s<\infty .
\end{gathered}
$$

Therefore from (18), we obtain

$$
\limsup _{t \rightarrow \infty} \int_{t_{0}}^{t} \delta^{2}(s) Q(s) \mathrm{d} s<\infty,
$$


which is a contradiction with (17). The proof is now complete.

Corollary 2.2. Assume that $\sigma(t) \leq t-\tau$ for $t \geq t_{0}$. In condition (2.1) and (17) hold, then every solution of Equation (1) is oscillatory.

Proof. The proof follows from Theorem 2.2.

To prove our next theorem, we need a class of function $\gamma$ and the operator $T$ defined as follows:

Following [16], we say that a function $\phi=\phi(t, s, \ell)$ belongs to the function class $Y$, denoted by $\phi \in Y$ if $\phi \in C(E, \mathbb{R})$, where $E=\left\{(t, s, \ell): t_{0} \leq \ell \leq s \leq t<\infty\right\}$, which satisfies $\phi(t, t, \ell)=0, \phi(t, \ell, \ell)=0$ and $\phi(t, s, \ell)>0$ for $\ell<s<t$, and has the partial derivative $\frac{\partial \phi}{\partial s}$ on $E$ such that $\frac{\partial \phi}{\partial s}$ is locally integrable with respect to $s$ in $E$.

Define the operator $T$ by

$$
T[g ; \ell, t]=\int_{\ell}^{t} \phi(t, s, \ell) g(s) \mathrm{d} s,
$$

for $t \geq s \geq \ell \geq t_{0}$ and $g \in C^{\prime}\left[t_{0}, \infty\right)$. The function $\psi=\psi(t, s, \ell)$ is defined by

$$
\frac{\partial \psi}{\partial s}(t, s, \ell)=\psi(t, s, \ell) \phi(t, s, \ell)
$$

then, it is easy to see that $T$ is a linear operator and

$$
T\left[g^{\prime} ; \ell, t\right]=-T[g \psi ; \ell, t] \text { for } g \in C^{\prime}\left[t_{0}, \infty\right) .
$$

Theorem 2.3. Assume that $\sigma(t) \leq t-\tau$, and there exist functions $\phi \in Y$ and $\rho \in C^{\prime}\left(\left[t_{0}, \infty\right), \mathbb{R}^{+}\right)$such that

$$
\limsup _{t \rightarrow \infty} T\left[k \rho(s) Q(s)-\frac{(1+a+b)\left(\psi+\frac{\rho^{\prime}(s)}{\rho(s)}\right)^{2}}{4 \sigma^{\prime}(s)} r(\sigma(s)) \rho(s) ; \ell, t\right]>0
$$

and

$$
\limsup _{t \rightarrow \infty} T\left[k Q(s)-\frac{(1+a+b) r(s) \psi^{2}}{4} ; \ell, t\right]>0
$$

where $Q(t)$ is defined as in Theorem 2.1, the operator $T$ defined by (19), and $\psi=\psi(t, s, \ell)$ is defined by (20). Then every solution of Equation (1) is oscillatory.

Proof. Let $x(t)$ be a nonoscillatory solution of Equation (1). Then there exists a $t_{1} \geq t_{0}$ such that $x(t) \neq 0$ for all $t \geq t_{1}$. Without loss of generality, we may assume that $x(t)>0, x(t-\tau)>0, x(t+\delta)>0$ and $x(\sigma(t))>0$ for all $t \geq t_{1}$. Then proceeding as in the proof of Theorem 2.1 we have $z(t)>0, z^{\prime}(t)>0,\left(r(t) z^{\prime}(t)\right)^{\prime} \leq 0$ or $z(t)>0, z^{\prime}(t)<0$ and $\left(r(t) z^{\prime}(t)\right)^{\prime} \leq 0$ for all $t \geq t_{1}$.

First assume that $z(t)>0, z^{\prime}(t)>0$, and $\left(r(t) z^{\prime}(t)\right)^{\prime} \leq 0$ for all $t \geq t_{1}$. Define

$$
w(t)=\rho(t) \frac{r(t) z^{\prime}(t)}{z(\sigma(t))}, t \geq t_{1} .
$$

Then $w(t)>0$, and

$$
\begin{aligned}
w^{\prime}(t) & =\rho(t) \frac{\left(r(t) z^{\prime}(t)\right)^{\prime}}{z(\sigma(t))}+\frac{\rho^{\prime}(t)}{\rho(t)} w(t)-\frac{w(t)}{z(\sigma(t))} z^{\prime}(\sigma(t)) \sigma^{\prime}(t) \\
& \leq \rho(t) \frac{\left(r(t) z^{\prime}(t)\right)^{\prime}}{z(\sigma(t))}+\frac{\rho^{\prime}(t)}{\rho(t)} w(t)-\frac{w^{2}(t)}{\rho(t) r(\sigma(t))} \sigma^{\prime}(t) .
\end{aligned}
$$


Since $r(t) z^{\prime}(t)$ is nonincreasing and $z(t)$ is increasing. Next, define

$$
u(t)=\rho(t) \frac{\left(r(t-\tau) z^{\prime}(t-\tau)\right)}{z(\sigma(t))}, t \geq t_{1}
$$

Then $u(t)>0$, and

$$
\begin{aligned}
u^{\prime}(t) & =\rho(t) \frac{\left(r(t-\tau) z^{\prime}(t-\tau)\right)}{z(\sigma(t))}+\frac{\rho^{\prime}(t)}{\rho(t)} u(t)-\frac{u(t)}{z(\sigma(t))} z^{\prime}(\sigma(t)) \sigma^{\prime}(t) \\
& \leq \rho(t) \frac{\left(r(t-\tau) z^{\prime}(t-\tau)\right)^{\prime}}{z(\sigma(t))}+\frac{\rho^{\prime}(t)}{\rho(t)} u(t)-\frac{u^{2}(t) \sigma^{\prime}(t)}{\rho(t) r(\sigma(t))} .
\end{aligned}
$$

Since $r(t) z^{\prime}(t)$ is nonincreasing, $z(t)$ is increasing and $\sigma(t) \leq t-\tau$. Again, define

$$
v(t)=\rho(t) \frac{\left(r(t+\delta) z^{\prime}(t+\delta)\right)}{z(\sigma(t))}, t \geq t_{1} .
$$

Then $v(t)>0$, and

$$
\begin{aligned}
v^{\prime}(t) & =\rho(t) \frac{\left(r(t+\delta) z^{\prime}(t+\delta)\right)}{z(\sigma(t))}+\frac{\rho^{\prime}(t)}{\rho(t)} v(t)-\frac{v(t)}{z(\sigma(t))} z^{\prime}(\sigma(t)) \sigma^{\prime}(t) \\
& \leq \rho(t) \frac{\left(r(t+\delta) z^{\prime}(t+\delta)\right)^{\prime}}{z(\sigma(t))}+\frac{\rho^{\prime}(t)}{\rho(t)} v(t)-\frac{v^{2}(t) \sigma^{\prime}(t)}{\rho(t) r(\sigma(t))} .
\end{aligned}
$$

Since $r(t) z^{\prime}(t)$ is nonincreasing, $z(t)$ is increasing and $\sigma(t) \leq t+\delta$. Combining (25) and (29), and then using (4), we obtain

$$
\begin{aligned}
w^{\prime}(t)+a u^{\prime}(t)+b v^{\prime}(t) \leq & -k \rho(t) Q(t)+\frac{\rho^{\prime}(t)}{\rho(t)} w(t)-\frac{\sigma^{\prime}(t)}{r(\sigma(t)) \rho(t)} w^{2}(t)+a \frac{\rho^{\prime}(t)}{\rho(t)} u(t) \\
& -a \frac{\sigma^{\prime}(t)}{r(\sigma(t)) \rho(t)} u^{2}(t)+b \frac{\rho^{\prime}(t)}{\rho(t)} v(t)-b \frac{\sigma^{\prime}(t)}{r(\sigma(t)) \rho(t)} v^{2}(t) .
\end{aligned}
$$

Now applying the operator $T$ to (30) and then using (21), we have

$$
\begin{aligned}
T[k \rho(s) Q(s) ; \ell, t] \leq & T\left[\left(\psi+\frac{\rho^{\prime}(s)}{\rho(s)}\right) w(s)-\frac{\sigma^{\prime}(s)}{r(\sigma(s)) \rho(s)} w^{2}(s)+a\left(\psi+\frac{\rho^{\prime}(s)}{\rho(s)}\right) u(s)\right. \\
& \left.-a \frac{\sigma^{\prime}(s)}{r(\sigma(s)) \rho(s)} u^{2}(s)+b\left(\psi+\frac{\rho^{\prime}(s)}{\rho(s)}\right) v(s)-b \frac{\sigma^{\prime}(s)}{r(\sigma(s)) \rho(s)} v^{2}(s) ; \ell, t\right] .
\end{aligned}
$$

From the last inequality, we obtain

$$
T[k \rho(s) Q(s) ; \ell, t] \leq T\left[\frac{(1+a+b)}{4 \sigma^{\prime}(s)}\left(\psi+\frac{\rho^{\prime}(s)}{\rho(s)}\right)^{2} r(\sigma(s)) \rho(s) ; \ell, t\right]
$$

or

$$
T\left[k \rho(s) Q(s)-\frac{(1+a+b)}{4 \sigma^{\prime}(s)}\left(\psi+\frac{\rho^{\prime}(s)}{\rho(s)}\right)^{2} r(\sigma(s)) k(s) ; \ell, t\right] \leq 0 .
$$

Taking the sup limit in the last inequality, we obtain a contradiction with (22).

Next consider the case $z(t)>0, z^{\prime}(t)<0$ and $\left(r(t) z^{\prime}(t)\right)^{\prime} \leq 0$ for all $t \geq t_{2}$. From the proof of Theorem 2.1, we have the inequality (16). Now apply the operator $T$ to (16) and then using (21), we have 


$$
T[k Q(s) ; \ell, t] \leq T\left[\psi w(s)-\frac{w^{2}(s)}{r(s)}+a \psi u(s)-a \frac{u^{2}(s)}{r(s)}+b \psi v(s)-b \frac{v^{2}(s)}{r(s)} ; \ell, t\right]
$$

From the last inequality, we obtain

$$
T[k Q(s) ; \ell, t] \leq T\left[\frac{(1+a+b) r(s) \psi^{2}}{4} ; \ell, t\right]
$$

or

$$
T\left[k Q(s)-\frac{(1+a+b)}{4} \psi^{2} r(s) ; \ell, t\right] \leq 0 .
$$

Taking the sup limit in the last inequality, we obtain a contradiction with (23). The proof is now completed.

Remark 2.1. With different choices of functions $\rho$ and $\phi$, Theorem 2.3 can be stated with different conditions for oscillations of Equation (1).

For example, if we take $\phi(t, s, \ell)=(t-s)^{\alpha}(s-\ell)^{\beta}$ for $\alpha>\frac{1}{2}, \beta>\frac{1}{2}$, then

$$
\psi(t, s, \ell)=\frac{\beta t-(\alpha+\beta) s+\alpha \ell}{(t-s)(s-\ell)} .
$$

From Theorem 2.3, we obtain the following oscillation criteria for Equation (1).

Corollary 2.3. Assume that $\sigma(t) \leq t-\tau$, and there exists a function $\rho \in C^{\prime}\left(\left[t_{0}, \infty\right), \mathbb{R}^{+}\right)$such that

$$
\limsup _{t \rightarrow \infty} \int_{t_{0}}^{t}\left[k(t-s)^{\alpha}\left(s-t_{0}\right)^{\beta} \rho(s) Q(s)-\frac{(1+a+b)\left(\psi+\frac{\rho^{\prime}(s)}{\rho(s)}\right)^{2}}{4 \sigma^{\prime}(s)} r(\sigma(s)) \rho(s)\right] \mathrm{d} s>0
$$

and

$$
\limsup _{t \rightarrow \infty} \int_{t_{0}}^{t}\left[k(t-s)^{\alpha}\left(s-t_{0}\right)^{\beta} Q(s)-\frac{(1+a+b) r(s) \psi^{2}}{4}\right] \mathrm{d} s>0
$$

where $\alpha>\frac{1}{2}, \beta>\frac{1}{2}$ and $\psi=\frac{\beta t-(a+b) s+\alpha t_{0}}{(t-s)\left(s-t_{0}\right)}$. Then every solution of Equation (1) is oscillatory.

\section{Examples}

In this section, we provide three examples to illustrate the main results.

Example 3.1. Consider the neutral differential equation

$$
\left(t^{2}(x(t)+3 x(t-\pi)+2 x(t+\pi))^{\prime}\right)+5 t x(t-\pi)=0, t \geq 1 .
$$

Here $r(t)=t^{2}, a(t)=3, b(t)=2, q(t)=5 t, \tau=\delta=\pi$, and $\sigma(t)=t-\pi$. By taking $n=1$ and $\delta(t)=\frac{1}{t}$, it is easy to see that all conditions of Theorem 2.1 are satisfied and hence every solution of Equation (31) is oscillatory.

Example 3.2. Consider the neutral differential equation

$$
\left(t^{2}(x(t)+2 x(t-2)+2 x(t+1))^{\prime}\right)+\frac{100}{(t-1)^{2}} x(t-3)=0, t \geq 4
$$


Here $r(t)=t^{2}, a(t)=2, b(t)=1, q(t)=\frac{100}{(t-1)^{2}}, \tau=2, \delta=1$, and $\sigma(t)=t-3$. By taking $\alpha=\beta=2$ and $\rho(t)=1$, it is easy to see that all conditions of Corollary 2.3 are satisfied and hence every solution of Equation (32) is oscillatory.

We conclude this paper with the following remark.

Remark 3.1. The results presented in [24] are not applicable to Equations (31) and (32) since in these equations $\int_{t_{0}}^{\infty} \frac{1}{r(t)} \mathrm{d} t<\infty$ and the neutral term contains advanced arguments. Therefore, our results complement and generalize some of the known results in the literature.

\section{References}

[1] Bainov, D.D. and Mishev, D.P. (1991) Oscillation Theory of Neutral Differential Equations with Delay. Adam Hilger, Bristol.

[2] Gyori, I. and Ladas, G. (1991) Oscillation Theory of Delay Differential Equations with Applications. Claredon Press, Oxford.

[3] Hale, J. (1977) Theory of Functional Differential Equations. Springer, New York. http://dx.doi.org/10.1007/978-1-4612-9892-2

[4] Agarwal, R.P. and Grace, S.R. (1999) Oscillation Theorems for Certain Neutral Functional Differential Equations. Computers \& Mathematics with Applications, 38, 1-11. http://dx.doi.org/10.1016/S0898-1221(99)00280-1

[5] Driver, R.D. (1984) A Mixed Neutral System. Nonlinear Analysis, 8, 155-158. http://dx.doi.org/10.1016/0362-546X(84)90066-X

[6] Dzurina, J., Busa, J. and Airyan, E.A. (2002) Oscillation Criteria for second Order Differential Equations of Neutral Type with Mixed Arguments. Differential Equation, 38, 137-140.

[7] Dzurina, J. and Stavroulakis, I.P. (2003) Oscillation Criteria for Second Order Delay Differential Equations. Applied Mathematics and Computation, 140, 445-453. http://dx.doi.org/10.1016/S0096-3003(02)00243-6

[8] Grace, S.R. (1992) Oscillation Theorems for Nonlinear Differential Equations of Second Order. Journal of Mathematical Analysis and Applications, 171, 220-241. http://dx.doi.org/10.1016/0022-247X(92)90386-R

[9] Grace, S.R. (1995) On the Oscillation of Mixed Neutral Equations. Journal of Mathematical Analysis and Applications, 194, 377-388. http://dx.doi.org/10.1006/jmaa.1995.1306

[10] Grace, S.R. (1995) Oscillation of Mixed Neutral Functional Differential Equations. Applied Mathematics and Computation, 68, 1-13. http://dx.doi.org/10.1016/0096-3003(94)00075-F

[11] Grammatikopouls, M.K., Ladas, G. and Meimaridou, A. (1985) Oscillation of Second Order Neutral Delay Differential Equations. Radovi Matematicki, 1, 267-274.

[12] Han, Z.L., Li, T.X., Sun, S.R. and Chen, W.S. (2010) On the Oscillation of Second Order Neutral Delay Differential Equations. Advances in Difference Equations, 2010, Article ID: 289340.

[13] Karpuz, B., Manojlovic, J.V., Ocalan, O. and Shoukaku, Y. (2009) Oscillation Criteria for a Class of Second Order Neutral Delay Differential Equations. Applied Mathematics and Computation, 210, 303-312. http://dx.doi.org/10.1016/j.amc.2008.12.075

[14] Li, T.X., Rogovchenko, Y.V. and Zhang, C.H. (2013) Oscillation of Second Order Neutral Differential Equations. Funkcialaj Ekvacioj, 56, 111-120. http://dx.doi.org/10.1619/fesi.56.111

[15] Li, T.X. and Thandapani, E. (2014) Oscillation of Solutions to Second Order Neutral Differential Equations. Electronic Journal of Differential Equations, 2014, 1-7.

[16] Liu, L.H. and Bai, Y.Z. (2009) New Oscillation Crieria for Second Order Nonlinear Neutral Delay Differential Equations. Journal of Computational and Applied Mathematics, 231, 657-663.

[17] Liu, X.Y. and Tang, X.H. (2007) Oscillation of Solutions of Neutral Differential Equations with a Superlinear Neutral Term. Applied Mathematics Letters, 20, 1016-1022. http://dx.doi.org/10.1016/j.aml.2006.11.006

[18] Ruan, S.G. (1993) Oscillations of Second Order Neutral Differential Equations. Canadian Mathematical Bulletin, 36, 485-496. http://dx.doi.org/10.4153/CMB-1993-064-4

[19] Sahina, Y. (2004) On Oscillation of Second Order Neutral Type Delay Differential Equations. Applied Mathematics and Computation, 150, 697-706. http://dx.doi.org/10.1016/S0096-3003(03)00300-X

[20] Sun, S.R., Li, T.X., Han, Z.L. and Zhang, C. (2013) On Oscillation of Second Order Nonlinear Neutral Functional 
Differential Equations. Bulletin of the Malaysian Mathematical Sciences Society, 36, 541-554.

[21] Xu, R. and Meng, F.W. (2007) Oscillation Crieria for Second Order Quasi-Linear Neutral Delay Differential Equations. Applied Mathematics and Computation, 192, 216-222. http://dx.doi.org/10.1016/j.amc.2007.01.108

[22] Ye, L.H. and Xu, Z.T. (2009) Oscillation Crieria for Second Order Quasilinear Neutral Delay Differential Equations. Applied Mathematics and Computation, 207, 388-396. http://dx.doi.org/10.1016/j.amc.2008.10.051

[23] Han, Z.L., Li, T.X., Sun, S.R. and Sun, Y.B. (2010) Remarks on the Paper [Appl. Math. Comput. 207(2009) 388-396]. Applied Mathematics and Computation, 215, 3998-4007. http://dx.doi.org/10.1016/j.amc.2009.12.006

[24] Arul, R. and Shobha, V.S. Oscillation of Second Order Neutral Differential Equations with Mixed Neutral Term. International Journal of Pure and Applied Mathematics. (To Appear) 